\title{
Escala de Estratégias de Aprendizagem: evidências de validade em contexto universitário híbrido
}

\author{
Lara Barros Martins - Universidade de São Paulo, Ribeirão Preto, Brasil \\ Thaís Zerbini - Universidade de São Paulo, Ribeirão Preto, Brasil
}

\begin{abstract}
Resumo
Instrumentos capazes de identificar e mensurar as estratégias de aprendizagem empregadas por alunos em cursos a distância ou híbridos assumem grande relevância, considerando a ampliação da modalidade. Objetivou-se adaptar e verificar as evidências de validade de um instrumento de estratégias de aprendizagem em instituição de ensino superior. Participaram do estudo, presencial ou virtualmente, alunos de uma universidade particular no interior paulista, que realizavam disciplinas semipresenciais. Foram realizadas análises fatoriais exploratórias e de consistência interna. A escala possui quatro fatores: estratégias cognitivas (15 itens, $\alpha=0,90)$; controle da emoção (4 itens, $\alpha=0,77)$; estratégias autorregulatórias (7 itens, $\alpha=0,86$ ); e busca de ajuda interpessoal ( 3 itens, $\alpha=0,68)$. Esta estrutura difere empiricamente das encontradas em estudos existentes com a medida. Sugere-se a sua aplicação em contextos e amostras diferentes, sobretudo, em ensino superior $100 \%$ a distância, em futuras pesquisas.

Palavras-chave: Educação a distância; Instituições de ensino superior; Estratégias de aprendizagem.
\end{abstract}

Scale of Learning Strategies: validity evidences in hybrid higher education context

\begin{abstract}
Instruments to identify and measure learning strategies used by students in distance and hybrid courses are very important, considering the expansion of e-learning. The objective was to adapt and verify the evidences of validity of an instrument of learning strategies in higher education institutions. Students of a private university in the state of São Paulo who were enroled in blended learning disciplines participated of this study, in person or remotely. Exploratory factor and internal consistency analisys were conducted. The scale has four factors: cognitive strategies ( 15 items, $\alpha=0.90)$, emotion control ( 4 items, $\alpha=0.77$ ), self-regulatory strategies (7items, $\alpha=0.86$ ) and interpersonal help seeking ( 3 items, $\alpha=0.68$ ). This structure differs empirically from those found in others studies with the scale. Future research should use it in different contexts and samples, especially in a $100 \%$ distance higher education course.

Keywords: Distance education; Higher education institutions; Learning strategies.
\end{abstract}

Escala de Estrategias de Aprendizaje: evidencias de validez en contexto universitario híbrido

\begin{abstract}
Resumen
Instrumentos para identificar y medir las estrategias de aprendizaje utilizadas por estudiantes en los cursos a distancia e híbridos son de gran importancia, teniendo en cuenta la expansión de la educación a distancia. El objetivo fue adaptar y verificar las evidencias de validez de un instrumento de estrategias de aprendizaje en una institución de enseñanza superior. Participaron del estudio, de forma presencial o virtual, estudiantes de una universidad privada del estado de São Paulo, que realizaban disciplinas semipresenciales. Fueron realizados análisis factoriales exploratorios y de confiabilidad. La escala tiene cuatro factores: estrategias cognitivas (15 ítems, $\alpha=0,90)$, control de las emociones ( 4 ítems, $\alpha=0,77)$, estrategias de autorregulación ( 7 ítems, $\alpha=0,86$ ) y búsqueda de ayuda interpersonal ( 3 ítems, $\alpha=0,68$ ). Esta estructura difiere empíricamente de las de otros estudios. En futuras investigaciones se sugiere la aplicación en otros contextos y muestras, especialmente en la enseñanza superior $100 \%$ a distancia. Palabras-clave: Educación a distancia; Instituciones de enseñanza superior; Estrategias de aprendizaje.
\end{abstract}

\section{Introdução}

Em contextos educacionais, os quais visam ao alcance da aprendizagem, os aprendizes utilizam procedimentos para facilitar a aquisição, armazenamento e posterior aplicação dos conhecimentos aprendidos, denominados de estratégias de aprendizagem. Estas servem como recursos para o processamento de informação, estando positivamente associadas com a aprendizagem bem-sucedida em estudos na área educacional e da psicologia organizacional, que enfocam as características pessoais do aprendiz (Oliveira, Boruchovitch \& Santos, 2009; Warr \& Downing, 2000).
Badia e Monereo (2010) definem as estratégias de aprendizagem como a tomada consciente e intencional de decisões, adaptadas às condições do contexto em que se realizará a ação e consistente na ativação de conhecimentos de natureza diversa, para alcançar um objetivo de aprendizagem. Dessa forma, cada aluno emprega diferentes procedimentos para conhecer, controlar e regular sua aprendizagem, não havendo uma melhor estratégia, mas alternativas mais eficazes, que variam de acordo com diferentes contextos de aprendizagem (Levin, 1986).

Os contextos virtuais promovem e requerem o desenvolvimento de habilidades para uma aprendizagem 
mais independente e flexível, considerando tempo, espaço e ritmo do aprendiz, por meio de um conjunto de recursos didáticos adaptados a tecnologias e múltiplas mídias (Carswell \& Venkatesh, 2002; Castro \& Ferreira, 2006; Cheung, 2000), sendo as estratégias de aprendizagem utilizadas nesses ambientes consideradas competências e habilidades imprescindíveis, auxiliando os aprendizes a planejar, regular e avaliar seus próprios processos de aprendizagem (Badia \& Monereo, 2010). Segundo esses autores, os ambientes instrucionais baseados em computadores têm sido apontados como úteis para o ensino de estratégias, pois possuem características singulares, como: a) necessidade de planejamento e revisão das decisões para alcançar os resultados almejados; b) promoção de uma interação dinâmica entre os objetos de conhecimento e os sujeitos que compartilham sua aquisição por meio das tecnologias de informação e comunicação (TICs); e c) possibilidades aumentadas de aprender novas formas de gestão do conhecimento graças à versatilidade dos formatos de representação da informação e à facilidade para criar e modificar redes de conhecimento.

As estratégias de aprendizagem e a inserção contínua das TICs em contextos educacionais acompanham o movimento recente de um novo paradigma tecnológico que se instaura e modifica tanto as práticas sociais quanto as educacionais (Badia \& Monereo, 2010; Coll \& Monereo, 2010; Zerbini, 2007). Nesse sentido, a pesquisa e inovação no ensino e na aprendizagem de estratégias em ambientes virtuais constituem um território a ser explorado, que deve atentar para os novos perfis e competências dos atores envolvidos, para os novos cenários, ferramentas e finalidades colocadas pelas novas modalidades de ensino. Estas, por serem mais personalizadas e autônomas, permitem que os aprendizes assumam cotas maiores de autoaprendizagem, e todas essas mudanças poderão influenciar os processos de aprendizagem.

As estratégias de aprendizagem são passíveis de serem aprendidas e, portanto, modificadas. Assim, o ensino e aperfeiçoamento de tais estratégias implicariam, não necessariamente, na mudança dos procedimentos instrucionais, mas afetariam a maneira que os aprendizes procedem ao aprender, podendo melhorar seu aproveitamento durante cursos a distância. O estudo das estratégias de aprendizagem pode orientar o planejamento instrucional de um curso e auxiliar no entendimento dos processos de aprendizagem individuais envolvidos (Santos, Boruchovitch, Primi, Zenorini \& Bueno, 2004; Zerbini, 2007).
Aprofundar o entendimento das estratégias de aprendizagem permitiria conhecer a competência das pessoas enquanto aprendizes e elucidar como elas podem aprender melhor como se aprende (Warr \& Allan, 1998). Investigações dedicadas a identificar quais estratégias são mais adequadas em cursos a distância, podem fornecer informações importantes para ampliar resultados de aprendizagem e serem ensinadas previamente à realização do curso. Apesar da possibilidade de intervenção permitida pelas estratégias de aprendizagem, em análise da literatura da área de ações educacionais presenciais e a distância, no período de 2000 a 2012, verifica-se um número reduzido de produções científicas nacionais e internacionais envolvendo tais características cognitivo-comportamentais.

Dentre os principais instrumentos disponíveis na literatura internacional para mensurar estratégias de aprendizagem em contextos escolares, como citado por Boruchovitch e cols. (2006) e Santos e cols. (2004), estão: o Learning and Study Strategies Inventory (LASSI) desenvolvido por Weinstein e Palmer (1987; 1990), o Motivated Strategies for Learning Questionnaire (MSLQ) de Pintrich e Groot (1989), e a Self-Regulated Learning Interview Schedule, de Zimmerman (1986), Zimmerman (1998) e Zimmerman e Martinez-Pons (1986).

Outros estudos nacionais e estrangeiros que propuseram instrumentos de medida para mensurar estratégias de aprendizagem enfatizam contextos escolares no nível fundamental (Boruchovitch \& Santos, 2004; Boruchovitch e cols., 2006; Costa \& Boruchovitch, 2009; Oliveira e cols., 2009) e nível superior de ensino (Bessa \& Tavares, 2001; Silva, 2004; Ribeiro \& Silva, 2007; Santos e cols., 2004). Há aqueles que enfocaram sobre as estratégias de aprendizagem usadas em ambientes de trabalho como cursos técnicos profissionalizantes (Warr \& Downing, 2000), treinamentos a distância (Zerbini, Carvalho \& Abbad, 2005) e cursos de qualificação profissional a distância (Borges-Ferreira, 2005; Zerbini \& Abbad, 2008).

Pesquisas na área da Educação, mais especificadamente em contexto escolar, têm demonstrado que o ensino de estratégias de aprendizagem aos alunos promove maior autonomia pessoal e aumenta sua consciência e responsabilidade sobre o próprio processo de aprendizagem e os resultados desses estudos convergem na relação positiva entre o uso adequado de estratégias e um melhor desempenho acadêmico (Costa $\&$ Boruchovitch, 2000; Santos e cols., 2004). Alertam, no entanto, sobre a escassez de pesquisas sobre estratégias de aprendizagem de universitários brasileiros. 
Algumas das escalas construídas e validadas com a finalidade de avaliar as estratégias de aprendizagem usadas por participantes de diferentes eventos instrucionais, que serão apresentadas a seguir, foram baseadas na definição teórica e trabalhos empíricos dos autores Warr e Allan (1998), realizados tanto em ambientes escolares como organizacionais. Estes autores definem as estratégias de aprendizagem como um conjunto de capacidades cognitivas, habilidades comportamentais e de autocontrole emocional utilizado pelo aprendiz para controlar os próprios processos psicológicos de aprendizagem, como atenção, aquisição, memorização e transferência, similar à conceituação de Badia e Monereo (2010), na qual o aluno assume um papel central e ativo no processo de aprendizagem ao escolher estratégias compatíveis a cada objetivo educacional específico a ser alcançado. São apontadas duas categorias principais de estratégias de aprendizagem: primárias (cognitivas e comportamentais) e autorregulatórias. As primeiras são usadas diretamente no momento de contato do aprendiz com o material a ser aprendido, tendo influências sobre seus processos de seleção, decodificação, armazenamento e recuperação. As autorregulatórias, por sua vez, têm um impacto indireto, influenciando a motivação do indivíduo para aprender, a autogestão de esforços e de automonitoramento do progresso durante a aprendizagem, e o controle de ansiedade diante de situações que poderiam dispersar a sua atenção.

A escala de Warr e Downing (2000) abordava as nove dimensões propostas por Warr e Allan (1998), a saber: a) cognitivas: repetição mental, organização e elaboração; b) comportamentais: busca de ajuda interpessoal, busca de ajuda ao material didático e aplicação prática; e c) autorregulatórias: controle da emoção, controle da motivação e monitoramento da compreensão. Além de aplicável em contexto escolar, é voltada especialmente para cursos técnicos, e foi aplicada a uma amostra composta por 152 adultos de um curso de mecânica básica. A versão final da escala contém 45 itens e oito fatores - dois fatores cognitivos "organização" e "elaboração" deram origem a um só, denominado "reflexão ativa".

Borges-Ferreira (2005), para avaliar a utilização de estratégias de aprendizagem por alunos de cursos a distância voltados à qualificação e formação profissionais, adaptou e revalidou a escala de estratégias de aprendizagem de Zerbini e cols. (2005). Este questionário foi parte integrante de um estudo com a finalidade, dentre outras, de avaliar as estratégias de aprendizagem utilizadas por participantes de treinamentos a distância e contava com 20 itens respondidos por 1.860 participantes - matriculados em um curso oferecido gratuitamente via web, que tinha como objetivo capacitar o aluno a elaborar um plano de negócios. O instrumento apresenta uma estrutura com três fatores: busca de ajuda interpessoal (8 itens, $\alpha=0,85$ ); elaboração e aplicação prática ( 5 itens, $\alpha=0,75)$; repetição, organização e ajuda do material (7 itens, $\alpha=0,78)$. No mesmo sentido, Borges-Ferreira (2005), para avaliar a utilização de estratégias de aprendizagem por alunos de cursos a distância voltados à qualificação e formação profissionais, adaptou e revalidou a escala de estratégias de aprendizagem de Zerbini e cols. (2005). Em uma amostra de 2.368 participantes, foi encontrada uma estrutura semelhante à das autoras: busca de ajuda interpessoal ( 6 itens; $a=0,87$ ), elaboração e aplicação prática do conteúdo (5 itens; $a=0,76)$ e repetição, organização e ajuda do material (8 itens; $\mathrm{a}=0,80$ ). Ambos os estudos não incluíram as estratégias de aprendizagem autorregulatórias que constam nos estudos de Warr e Downing (2000) e Warr e Allan (1998), em razão das especificidades dos cursos avaliados, de natureza primordialmente cognitiva e de baixa complexidade.

Em seguida, defendendo a importância de se incluir as estratégias de aprendizagem em estudos nos contextos de organização e trabalho, Zerbini e Abbad (2008) avaliaram as estratégias de aprendizagem utilizadas por participantes do curso "Iniciando um Pequeno Grande Negócio” (IPGN) do Serviço Brasileiro de Apoio às Micro e Pequenas Empresas (SEBRAE), com uma população de 4.719 inscritos. O IPGN consistia em uma ação de qualificação profissional, ofertada a distância, com características de aprendizagem aberta e que utilizava a internet como principal meio de entrega.

A escala de Estratégias de Aprendizagem utilizada por Zerbini e Abbad (2008) apresenta 28 itens e 8 fatores, a saber: controle da emoção, busca de ajuda interpessoal, repetição e organização, controle da motivação, elaboração, busca de ajuda ao material didático e monitoramento da compreensão (respectivas cargas fatoriais e alfas dos fatores são mostrados adiante, na Tabela 1). Embora o trabalho das autoras pretendesse construir itens relativos às estratégias de aprendizagem autorregulatórias para verificar evidências de validade de um novo instrumento, tal objetivo não foi cumprido. A importância de incluí-las no instrumento refere-se ao fato de que tais estratégias, embora pouco estudadas (Warr \& Allan, 1998), parecem ser diferenciais no 
Tabela 1. Resumo das informações sobre o instrumento utilizado na pesquisa

\begin{tabular}{llcccc}
\hline Instrumento & Fatores & $\begin{array}{c}N^{o} \text { de } \\
\text { itens }\end{array}$ & Alfa & \multicolumn{2}{c}{ Cargas fatoriais } \\
\cline { 4 - 6 } & & 5 & 0,89 & 0,48 & 0,91 \\
\hline Estratégias de aprendizagem & Controle da emoção & 6 & 0,89 & 0,66 & 0,83 \\
(Zerbini \& Abbad, 2008) & Busca de ajuda interpessoal & 5 & 0,77 & $-0,35$ & $-0,77$ \\
& Repetição e organização & 4 & 0,84 & 0,69 & 0,86 \\
& Controle da motivação & 3 & 0,83 & 0,54 & 0,91 \\
& Elaboração & 2 & 0,75 & 0,68 & 0,82 \\
& Busca de ajuda ao material didático & 2 & 0,82 & $-0,49$ & $-0,75$ \\
& Monitoramento da compreensão & 3 & & &
\end{tabular}

apoio ao processo de ensino-aprendizagem em cursos a distância, viabilizando o autogerenciamento da aprendizagem necessário para que o aluno possa ser bem-sucedido em seus estudos.

A análise das pesquisas descritas acerca do uso das estratégias de aprendizagem permite concluir que, apesar do aumento crescente de cursos oferecidos a distância, fazem referência majoritariamente ao contexto presencial, tanto no campo da Educação como em ambientes profissionais. São necessários, portanto, mais estudos que mostrem resultados de eventos educacionais ofertados nessa modalidade, para levantar um panorama sobre as diferentes estratégias que os alunos utilizam para se beneficiar de tais cursos. Assim, a construção de instrumentos capazes de identificar e mensurar as estratégias de aprendizagem empregadas na Educação a Distância (EAD) assume grande relevância.

Dessa forma, o presente artigo pretende contribuir com a área de avaliação de sistemas instrucionais a distância ao adaptar e verificar evidências de validade do instrumento de medida Estratégias de Aprendizagem (Zerbini \& Abbad, 2008), em contexto de instituição de ensino superior (IES).

\section{Método}

\section{Participantes}

Participaram do estudo alunos que cursavam as disciplinas semipresenciais e obrigatórias Metodologia Científica $(\mathrm{N}=916)$ e Economia $(\mathrm{N}=433)$, que compunham a grade curricular de todos os cursos de graduação de uma universidade particular do interior paulista, a qual oferece, além dos tradicionais cursos presenciais, cursos a distância, via internet, desde 2008.
Os dados de 343 alunos das disciplinas mencionadas, que responderam ao questionário sociodemográfico, mostram que a maioria era do sexo feminino $(62,7 \%)$, solteira $(87,5 \%)$, sem filhos $(91,3 \%)$ e residente da região sudeste $(89,5 \%)$. Possuíam, em média, 23 anos de idade $(\mathrm{DP}=7,26)$ - faixa etária preponderante entre 17 e 20 anos $(46,6 \%)$ - e ensino superior incompleto $(65,9 \%)$. Muitos alunos apenas estudavam $(62,1 \%)$ e outros, além dos estudos, se dedicavam a atividades profissionais $(35,3 \%)$, estando a renda concentrada entre 2 e 3 salários mínimos (40,5\%). Grande parte dos alunos $(87,5 \%)$ possuía experiência anterior no uso da internet, indicando uma preparação anterior para utilizar ferramentas e recursos web.

Para os processos de validação semântica e por juízes da escala de Estratégias de Aprendizagem, participaram, respectivamente, quatro estudantes de graduação com características semelhantes aos da amostra e pesquisadores doutores $(n=3)$ e mestre $(n=1)$.

\section{Instrumento}

As informações sobre o instrumento de Estratégias de Aprendizagem, com 28 itens, que descrevem comportamentos utilizados pelos alunos no momento de estudo, ou seja, as estratégias de aprendizagem adotadas para estudar, estão associadas a uma escala tipo Likert de frequência de 11 pontos $(0=$ nunca a $10=$ sempre), utilizada na presente investigação e podem ser visualizadas na Tabela 1 .

Tal escala sofreu alterações para adequar-se às especificidades de contexto universitário e foi acrescida de itens de estratégias autorregulatórias, atendendo a recomendações sobre a probabilidade de que ações educacionais ofertadas a distância, via internet, exigem de seus usuários maior autonomia e autogerenciamento 
da aprendizagem para persistir nos estudos do que cursos presenciais (Zerbini, 2007).

\section{Procedimentos de coleta e análise de dados}

Previamente à aplicação dos questionários, o instrumento de Estratégias de Aprendizagem adaptado (41 itens) passou por validação semântica e por juízes. Na primeira, alunos de graduação avaliaram cada item quanto à precisão, clareza e objetividade, bem como quanto à adequação das instruções da escala; o segundo processo consistiu na ponderação por especialistas sobre a adequação do instrumento produzido (revisão dos itens, análise da necessidade de incluir ou excluir itens, apontamento do fator correspondente a cada item).

Foram necessários três momentos diferentes para a coleta de dados visando aumentar os índices de retorno dos questionários respondidos. Os dois primeiros momentos da coleta foram realizados a distância, por meio da internet, pela qual o instrumento de Estratégias de Aprendizagem digitalizado estava disponível no ambiente virtual de aprendizagem (AVA); no terceiro momento de coleta, optou-se pela aplicação presencial, em papel, dos questionário. Portanto, o instrumento foi disponibilizado três vezes aos alunos das disciplinas da IES parceira $(\mathrm{N}=1349)$, tendo obtido um índice de retorno total de respostas de $22,2 \%(n=300)$. As respostas ao questionário integraram o arquivo de dados no SPSS (Statistical Package for the Social Science) versão 17.0 - software que possibilitou as análises do material obtido.

Foram feitas análises descritivas e exploratórias para investigar a exatidão da entrada dos dados, a presença de casos extremos, a distribuição dos casos omissos, a distribuição de frequência das variáveis e o tamanho das amostras - conforme orientações de Tabachnick e Fidell (2001). Para verificar as evidências de validade do instrumento foi calculado o índice de consistência interna (alfa de Cronbach) e realizadas análises exploratórias fatoriais, por meio da análise dos componentes principais (Principal Components - PC) e do método de fatoração dos eixos principais (Principal Axis Factoring - $P A F)$. A $P C$ visa reduzir o número de variáveis em componentes, que expliquem a maior parte da variância original das variáveis, além de analisar os componentes mais importantes, definindo o número mínimo de fatores a serem inicialmente extraídos. A $P A F$ verifica a covariância entre as variáveis, ou seja, a variabilidade que as variáveis possuem em comum, indicando o número de fatores existentes - tendo em conta os componentes previamente apontados pela $P C$.

\section{Resultados e discussão}

Os processos de validação semântica e por juízes contribuíram para aperfeiçoar a redação de alguns itens, que sofreram pequenas alterações e, principalmente, para verificar a adequação do instrumento às ações educacionais ofertadas a distância, especificadamente, a contextos de ensino superior. Após os ajustes sugeridos nos processos de validação, novos itens referentes às estratégias de aprendizagem autorregulatórias foram incluídos - com base na escala originalmente desenvolvida por Warr e Downing (2000) - e outros foram excluídos, cumprindo-se o objetivo de adaptação do instrumento em questão, que ficou com 31 itens.

Em decorrência da primeira aplicação da escala em contexto de graduação a distância, e com vistas a explorar a medida, considerou-se de extrema importância verificar como os itens pertencentes às diferentes dimensões se comportariam e, do mesmo modo, averiguar a importância e a contribuição das estratégias cognitivas, comportamentais e autorregulatórias no momento de estudo para alunos do nível superior de ensino.

O Questionário de Estratégias de Aprendizagem apresentou 299 casos válidos e 44 casos omissos em 30 de seus 31 itens - no item 7, em decorrência de um erro não recuperado no sistema utilizado na coleta de dados, havia 96 casos omissos. Não foram estimados valores para substituir os dados ausentes, pois estes resultam do não preenchimento de questionários inteiros pelos participantes, não havendo, portanto, subgrupos amostrais. As respostas não apresentaram casos extremos univariados, mas 21 casos extremos multivariados foram identificados e retirados do arquivo de dados, totalizando 278 casos válidos sem outliers multivariados.

A partir da elaboração e análise da matriz de covariância verificou-se que não houve relacionamentos não lineares entre as variáveis (linearidade), nem a presença de singularidade e multicolinearidade. Em termos de fatorabilidade, foram analisados o tamanho das correlações e a adequação da amostra. Em mais de 50\% dos casos foram encontrados valores de correlação superiores a 0,30 , indicando que a matriz provavelmente era fatorizável. Quanto ao teste de Kaiser-Meyer-Olkin (KMO), obteve-se um valor de 0,87 , considerado um excelente índice de adequação da amostra.

A extração inicial de fatores foi feita mediante análise dos componentes principais, seguindo os critérios convencionais. A análise dos componentes principais, com tratamento listwise para os casos omissos, sugere 
uma estrutura empírica com 8 componentes que explicam, em conjunto, $66,08 \%$ da variância total das respostas dos participantes aos itens do questionário - segundo critério dos eigenvalues (valores próprios) maiores ou iguais a 1 (um). Quanto à importância do fator, cada componente deveria explicar no mínimo 3\% da variância total - segundo critério de Harman, um fator é considerado irrelevante quando explica menos do que $3 \%$ da variância total das variáveis - assim, poderiam ser extraídos, no máximo, oito fatores. A análise do scree plot (distribuição visual dos valores próprios), em contrapartida, indicou a existência de 5 a 6 componentes. A análise paralela de Horn retifica o número de componentes indicado pela análise dos valores próprios, variância explicada e scree plot, apontando 4 componentes para este instrumento - tal análise compara valores próprios empíricos, obtidos pela análise da $P C$, com valores próprios aleatórios, em função da quantidade de variáveis e do tamanho da amostra. Apenas os componentes com valores empíricos maiores ou iguais aos valores aleatórios são mantidos na estrutura (Laros, 2004).

O método de rotação oblíqua e tratamento listwise para casos omissos foram utilizados para a fatoração dos eixos principais $(P A F)$. Foram incluídos na escala apenas os itens com conteúdos semânticos similares e cargas fatoriais superiores ou iguais a 0,30. Dessa análise foram extraídos 4 fatores que explicam, respectivamente, $31,06 \%, 5,51 \%, 4,33 \%$ e 3,38\% da variância total das respostas aos itens do instrumento. A opção pela solução com 4 fatores faz sentido empírico e também teórico.

A Tabela 2 apresenta a estrutura empírica da escala, as cargas fatoriais, as comunalidades $\left(h^{2}\right)$ dos itens, as médias e desvios padrão, os índices de consistência interna e os valores próprios e percentuais de variância explicada de cada fator.

Os resultados das análises exploratórias da escala não indicaram itens altamente correlacionados entre si. A Escala de Estratégias de Aprendizagem ficou com 29 itens e é formada por 4 fatores denominados: 1) "estratégias cognitivas", 2) "controle da emoção", 3) "estratégias autorregulatórias" e 4) "busca de ajuda interpessoal.

O fator 1, estratégias cognitivas, apresenta um total de 15 itens (16 ao 31, com exceção do item 19 que não permaneceu na estrutura) e possui um excelente índice de consistência interna $(\alpha=0,90)$, com cargas fatoriais variando entre 0,33 e 0,72 . Dos 15 itens pertencentes a esse fator, 12 são provenientes das estratégias cognitivas de repetição, organização e elaboração (reflexão ativa), dois itens provêm das estratégias de busca de ajuda ao material didático e um de aplicação prática, ambas comportamentais, segundo Warr e Allan (1998). Neste estudo, as estratégias cognitivas e algumas comportamentais se agruparam em um mesmo fator, assim como ocorreu nos estudos de Zerbini e cols. (2005), Borges-Ferreira (2005) e Silva (2004). É bastante coerente o agrupamento desses itens em um único fator, pois os exercícios propostos pelas disciplinas avaliadas medem apenas a compreensão de conteúdos e não sua aplicação prática. Assim, o item 19, "Realizei os exercícios práticos propostos ao longo do curso para me ajudar a aprender", não permaneceu na estrutura empírica.

Já o item 18, “Tentei entender o conteúdo ao aplicá-lo na prática, ao invés de dedicar tempo lendo ou pedindo ajuda a alguém", juntou-se às estratégias cognitivas. Como a maioria dos estudantes da amostra usa mais estratégias cognitivas durante o curso para dar conta dos conteúdos e exercícios propostos, talvez não estivesse sensível à identificação e/ou distinção dessa estratégia de "aplicação prática", que se aproxima das estratégias de organização, das demais, cognitivas. Do mesmo modo, não houve a diferenciação entre os itens das estratégias cognitivas (repetição, organização e elaboração), que foram entendidos como um conjunto similar de estratégias usadas no momento de estudo. Em contexto de graduação, tais estratégias foram tomadas em conjunto, sendo elas bastante comuns e mais usadas durante toda a vida acadêmica, já que os conteúdos são em sua maioria teóricos, e não práticos diferente do que ocorreu no estudo de Warr e Downing (2000), em que havia a delimitação, pois os autores avaliaram cursos presenciais em mecânica básica e cursos técnicos; ambos exigiam, em contrapartida, muita aplicação prática do conteúdo aprendido. Além disso, em decorrência da complexidade dos conteúdos, objetivos e exercícios propostos, talvez não tenha sido necessário o uso de estratégias de elaboração.

Quanto aos itens de busca de ajuda ao material didático terem se juntado a esse fator, é interessante notar que eles se referem à busca de fontes externas ao curso para auxiliar na aprendizagem: "Busquei outras fontes de pesquisa, fora da internet, relacionadas ao curso para me ajudar a aprender" e "Busquei sites relacionados ao conteúdo do curso para me ajudar a aprender". Do mesmo modo que o item de aplicação prática discutido, esses dois itens também se assemelham às estratégias de organização. Embora as estratégias exprimam um comportamento, está implícita a decisão (cognitiva) e 
Tabela 2. Estrutura empírica da Escala de Estratégias de Aprendizagem

\begin{tabular}{|c|c|c|c|c|c|c|c|}
\hline \multirow[t]{2}{*}{ Descrição dos itens } & \multicolumn{4}{|c|}{ Cargas fatoriais } & \multirow[t]{2}{*}{$\mathrm{h}^{2}$} & \multirow[t]{2}{*}{$\mathrm{X}$} & \multirow[t]{2}{*}{$\mathrm{DP}$} \\
\hline & 1 & 2 & 3 & 4 & & & \\
\hline 1. Mantive a calma quando tive dificuldades. & & 0,70 & & & 0,52 & 6,77 & 2,20 \\
\hline $\begin{array}{l}\text { 2. Repeti a mim mesmo, quando me senti ansioso, que tudo } \\
\text { sairia bem ao final do curso. }\end{array}$ & & 0,46 & & & 0,31 & 7,15 & 2,47 \\
\hline $\begin{array}{l}\text { 3. Mantive a calma com a possibilidade de ter um rendimento } \\
\text { abaixo do esperado. }\end{array}$ & & 0,76 & & & 0,58 & 5,73 & 2,53 \\
\hline $\begin{array}{l}\text { 4. Mantive a calma diante dos erros que cometi ao realizar } \\
\text { atividades do curso. }\end{array}$ & & 0,60 & & & 0,47 & 6,19 & 2,36 \\
\hline $\begin{array}{l}\text { 5. Esforcei-me mais quando percebi que estava perdendo a } \\
\text { concentração. }\end{array}$ & & & $-0,63$ & & 0,48 & 7,84 & 1,71 \\
\hline $\begin{array}{l}\text { 6. Forcei-me a manter a atenção nos estudos quando me } \\
\text { senti desinteressado. }\end{array}$ & & & $-0,65$ & & 0,49 & 7,36 & 1,95 \\
\hline $\begin{array}{l}\text { 7. Esforcei-me mais quando percebi que estava perdendo o } \\
\text { interesse no assunto. }\end{array}$ & & & $-0,61$ & & 0,48 & 7,37 & 1,69 \\
\hline $\begin{array}{l}\text { 8. Elaborei perguntas para testar minha compreensão sobre } \\
\text { os conteúdos do curso. }\end{array}$ & & & & & 0,31 & 5,46 & 2,73 \\
\hline $\begin{array}{l}\text { 9. Revisei a matéria para verificar o quanto eu dominava o } \\
\text { conteúdo. }\end{array}$ & & & $-0,50$ & & 0,43 & 7,01 & 2,34 \\
\hline $\begin{array}{l}\text { 10. Esforcei-me para verificar minha compreensão sobre o } \\
\text { que estava sendo ensinado. }\end{array}$ & & & $-0,69$ & & 0,58 & 7,46 & 1,87 \\
\hline $\begin{array}{l}\text { 11. Busquei auxílio do tutor para esclarecer minhas dúvidas } \\
\text { sobre o conteúdo. }\end{array}$ & & & & 0,45 & 0,30 & 4,86 & 3,20 \\
\hline $\begin{array}{l}\text { 12. Busquei auxílio de colegas nos fóruns para esclarecer } \\
\text { minhas dúvidas. }\end{array}$ & & & & 0,69 & 0,48 & 5,73 & 3,11 \\
\hline $\begin{array}{l}\text { 13. Troquei mensagens com os colegas para esclarecer dúvidas } \\
\text { sobre o conteúdo do curso. }\end{array}$ & & & & 0,51 & 0,30 & 5,96 & 3,10 \\
\hline $\begin{array}{l}\text { 14. Busquei solucionar minhas dúvidas ao consultar as } \\
\text { apostilas do curso. }\end{array}$ & & & $-0,67$ & & 0,48 & 7,71 & 2,08 \\
\hline $\begin{array}{l}\text { 15. Busquei compreender melhor os conteúdos ao estudá-los } \\
\text { nas apostilas do curso. }\end{array}$ & & & $-0,65$ & & 0,53 & 7,70 & 1,95 \\
\hline $\begin{array}{l}\text { 16. Busquei outras fontes de pesquisa, fora da internet, } \\
\text { relacionadas ao curso, para me ajudar a aprender. }\end{array}$ & 0,33 & & & & 0,20 & 6,68 & 3,06 \\
\hline $\begin{array}{l}\text { 17. Busquei sites relacionados ao conteúdo do curso para me } \\
\text { ajudar a aprender. }\end{array}$ & 0,44 & & & & 0,32 & 7,36 & 2,57 \\
\hline $\begin{array}{l}\text { 18. Tentei entender o conteúdo ao aplicá-lo na prática, ao } \\
\text { invés de dedicar tempo lendo ou pedindo ajuda a alguém. }\end{array}$ & 0,42 & & & & 0,25 & 6,00 & 2,55 \\
\hline $\begin{array}{l}\text { 19. Realizei os exercícios práticos propostos ao longo do curso } \\
\text { para me ajudar a aprender. }\end{array}$ & & & & & 0,26 & 7,26 & 2,49 \\
\hline $\begin{array}{l}\text { 20. Revisei os conteúdos relativos aos exercícios em que } \\
\text { cometi erros. }\end{array}$ & 0,37 & & & & 0,46 & 6,46 & 2,51 \\
\hline $\begin{array}{l}\text { 21. Aprendi conteúdos ao mentalizá-los repetidamente até } \\
\text { perceber que havia entendido. }\end{array}$ & 0,49 & & & & 0,46 & 6,48 & 2,58 \\
\hline $\begin{array}{l}\text { 22. Repeti mentalmente os conteúdos do curso que gostaria } \\
\text { de aprender. }\end{array}$ & 0,52 & & & & 0,51 & 6,28 & 2,66 \\
\hline
\end{tabular}


Tabela 2. Estrutura empírica da Escala de Estratégias de Aprendizagem (Continuação)

\begin{tabular}{|c|c|c|c|c|c|c|c|}
\hline \multirow[t]{2}{*}{ Descrição dos itens } & \multicolumn{4}{|c|}{ Cargas fatoriais } & \multirow[t]{2}{*}{$\mathrm{h}^{2}$} & \multirow[t]{2}{*}{$\mathrm{X}$} & \multirow[t]{2}{*}{$\mathrm{DP}$} \\
\hline & 1 & 2 & 3 & 4 & & & \\
\hline 23. Fiz anotações sobre o conteúdo do curso. & 0,54 & & & & 0,48 & 7,32 & 2,59 \\
\hline 24. Fiz resumos do conteúdo do curso. & 0,50 & & & & 0,48 & 6,78 & 2,87 \\
\hline $\begin{array}{l}\text { 25. Li o conteúdo do curso várias vezes como método para } \\
\text { aprender. }\end{array}$ & 0,37 & & & & 0,47 & 7,03 & 2,46 \\
\hline $\begin{array}{l}\text { 26. Fiz esquemas do conteúdo do curso como método para } \\
\text { aprender. }\end{array}$ & 0,53 & & & & 0,54 & 6,44 & 2,62 \\
\hline $\begin{array}{l}\text { 27. Refleti sobre as implicações que os conteúdos aprendidos } \\
\text { poderiam ter. }\end{array}$ & 0,66 & & & & 0,55 & 6,46 & 2,42 \\
\hline $\begin{array}{l}\text { 28. Busquei desenvolver uma ideia global sobre como os } \\
\text { conteúdos do curso se relacionavam entre si. }\end{array}$ & 0,67 & & & & 0,54 & 6,45 & 2,49 \\
\hline $\begin{array}{l}\text { 29. Associei os conteúdos do curso aos meus conhecimentos } \\
\text { anteriores. }\end{array}$ & 0,72 & & & & 0,48 & 7,38 & 2,10 \\
\hline $\begin{array}{l}\text { 30. Diferenciei, ao analisar os conteúdos do curso, os aspectos } \\
\text { mais importantes dos menos importantes. }\end{array}$ & 0,66 & & & & 0,46 & 6,90 & 1,98 \\
\hline $\begin{array}{l}\text { 31. Identifiquei situações diárias em que eu pudesse aplicar os } \\
\text { conteúdos do curso. }\end{array}$ & 0,60 & & & & 0,42 & 7,08 & 2,27 \\
\hline $\mathrm{N}$ & 278 & 278 & 226 & 278 & & & \\
\hline Eigenvalue (Valor próprio) & 9,62 & 1,70 & 1,34 & 1,05 & & & \\
\hline \% da variância explicada & 31,06 & 5,51 & 4,33 & 3,38 & & & \\
\hline $\mathrm{N}^{\circ}$ de itens & 15 & 4 & 7 & 3 & & & \\
\hline $\operatorname{Alfa}(\alpha)$ & 0,90 & 0,77 & 0,86 & 0,68 & & & \\
\hline
\end{tabular}

necessidade de outros materiais complementares aos conteúdos disponíveis. Assim, as estratégias deixam de ser comportamentais e passam a ser cognitivas. A identificação da provável insuficiência do material fornecido e/ou aliado à curiosidade por novos conhecimentos está expressa em um mesmo fator. Todos os conteúdos disponibilizados pelo curso e as fontes externas são virtuais, não impressas, similaridade que pode ter contribuído para juntar esses itens. Como o material do curso é virtual, no momento de estudo, o aluno simultaneamente busca outras fontes, também na internet, para complementar o entendimento dos conteúdos. Isso decorre da presença constante das TICs no dia a dia das pessoas e das características dos alunos contemporâneos, que estão permanentemente "plugados".

As estratégias de aprendizagem cognitivas têm se comportado de maneiras diferentes, dependendo do contexto de aplicação da escala, das características do curso e da amostra. Em Warr e Downing (2000), as estratégias de repetição distinguiram-se das de organização e elaboração, que compuseram o fator denominado de reflexão ativa (10 itens, $\alpha=0,90$, cargas fatoriais entre $0,47$ e 0,75$)$. E no estudo de Zerbini e Abbad (2008), repetição e organização ( 5 itens, $\alpha=0,77$, cargas fatoriais entre $-0,35$ e $-0,77)$ juntaram-se em um mesmo fator, separadas das estratégias de elaboração ( 3 itens, $\alpha=0,83$, cargas fatoriais entre 0,54 e 0,91 ).

O fator 2, controle da emoção, composto por 4 itens (1 ao 4), apresenta um índice razoável de consistência interna $(\alpha=0,77)$, com cargas fatoriais variando entre 0,46 e 0,76 . Assemelha-se aos fatores homônimos obtidos por Warr e Downing (2000) e por Zerbini e Abbad (2008). Nestes, o fator é composto por 5 itens, com índice de consistência interna de 0,85 e 0,89 , e cargas fatoriais de 0,48 a 0,91 e 0,66 a 0,72 , respectivamente. Os 4 itens permaneceram em um mesmo fator, como no estudo de Zerbini (2007), exprimindo estratégias que demonstram o controle da ansiedade e prevenção de dispersões de concentração causadas por sentimentos de ansiedade no momento de estudo, de acordo com Warr e Allan (1998). A permanência das mesmas na estrutura, em um fator distinto das demais 
estratégias, aponta para a importância de terem sido incluídas no instrumento, mostrando que possuem contribuição para o aprendizado de alunos que estudam a distância e, ademais, configuram um diferencial para o autogerenciamento da aprendizagem em contextos de EAD.

O fator 3, estratégias autorregulatórias, possui 7 itens $(5,6,7,9,10,14$ e 15), bom índice de consistência interna $(\alpha=0,86)$ e cargas fatoriais entre $-0,50$ e - 0,69 e é composto pelas estratégias de monitoramento da compreensão, controle da motivação e dois itens de busca de ajuda ao material didático. As estratégias autorregulatórias de controle da motivação e monitoramento da compreensão, propostas por Warr e Allan (1998), uniram-se em um mesmo fator. O controle da motivação e atenção atrelado à avaliação do processo de aquisição de aprendizagem parecem ocorrer em conjunto, não sendo identificados como distintos pela ótica dos estudantes. Tais estratégias auxiliam na autorregulação da aprendizagem (Boruchovitch \& Santos, 2004), portanto é cabível estarem juntas em um mesmo fator.

Ainda nesse fator, 2 itens originais de busca de ajuda ao material didático, "Busquei solucionar minhas dúvidas ao consultar as apostilas do curso" e "Busquei compreender melhor os conteúdos ao estudá-los nas apostilas do curso" se agruparam. Esses itens referem-se especificadamente ao material do curso: as apostilas. Considerando que o único material impresso disponibilizado em formato das tradicionais apostilas sejam os guias das disciplinas, é coerente pensar que tais itens tenham se juntado a estratégias autorregulatórias. Os guias fornecem informações, orientações e instruções pertinentes ao aluno sobre o uso do material e desenvolvimento da disciplina, como: objetivos educacionais; atividades de aprendizagem a serem cumpridas e seus respectivos prazos; datas das avaliações, notas e os seus critérios; programa e cronograma da disciplina. Os alunos, portanto, devem usar os guias para organizar seus estudos; consultar exemplos de como resolver as atividades/exercícios e participar dos fóruns; e checar se os objetivos educacionais foram alcançados. Isso pode configurar no monitoramento do aprendizado e na modificação do comportamento quando necessário - definição de monitoramento da compreensão, segundo Warr e Allan (1998). Portanto, esses 2 itens expressam estratégias comportamentais que auxiliam no monitoramento da compreensão e autorregulação da aprendizagem, tendo consideradas as particularidades do material avaliado.
O item 8, "Elaborei perguntas para testar minha compreensão sobre os conteúdos do curso", originário do fator monitoramento da compreensão, não permaneceu na escala. Talvez decorra do fato de ser impreciso com relação à ação "elaborar perguntas": elaborar perguntas mentalmente? Ou criar perguntas e respondê-las para testar a compreensão? Essa estratégia parece não ser muito utilizada pelos alunos ou pode ser uma estratégia mais adequada a conteúdos mais complexos.

Nos estudos de Warr e Downing (2000) e Zerbini e Abbad (2008), foram obtidos 3 fatores isolados (respectivos número de itens, consistência interna e cargas fatoriais encontrados nessas pesquisas estão entre parênteses): monitoramento da compreensão (5 itens, $\alpha=0,85$, cargas fatoriais entre 0,48 e 0,68 ; 3 itens, $\alpha=0,82$, cargas fatoriais entre $-0,49$ e $-0,75)$, controle da motivação (5 itens, $\alpha=0,89$, cargas fatoriais entre 0,64 e 0,$78 ; 4$ itens, $\alpha=0,84$, cargas fatoriais entre 0,69 e 0,86 ) e busca de ajuda ao material didático ( 5 itens, $\alpha=0,89$, cargas fatoriais entre 0,73 e 0,$80 ; 2$ itens, $\alpha=0,75$, cargas fatoriais entre 0,68 e 0,82$)$. Na revalidação da escala de Zerbini e cols. (2005) por Borges-Ferreira (2005), foi encontrada uma estrutura tridimensional composta pelos fatores busca de ajuda interpessoal ( 6 itens, $\alpha=0,87)$, estratégias de elaboração e aplicação prática (5 itens, $\alpha=0,76)$ e estratégias de repetição, organização e ajuda ao material ( 8 itens, $\alpha=0,80)$ - as estratégias autorregulatórias não foram incluídas no instrumento devido à natureza e à baixa complexidade dos cursos avaliados.

O fator 4, busca de ajuda interpessoal, possui 3 itens (11, 12 e 13), índice razoável de consistência interna $(\alpha=0,68)$ e cargas fatoriais variando entre 0,45 e 0,69. Os itens desse fator expressam a busca ativa, por iniciativa do próprio aluno, pela ajuda de outras pessoas (tutores ou pares) para tirar dúvidas. Esse fator foi encontrado em Zerbini e Abbad (2008) - 6 itens, $\alpha=0,89$, cargas fatoriais entre 0,66 e 0,83 - e em Warr e Downing (2000) -5 itens, $\alpha=0,84$, cargas fatoriais entre 0,54 e 0,68. Segundo Warr e Allan (1998), tais estratégias comportamentais representam um comportamento proativo do indivíduo de buscar auxílio, ao invés de obter informações do material do curso. A análise dos resultados descritivos mostra que a maior ocorrência de médias baixas concentra-se nos itens desse fator, ou seja, os alunos utilizaram pouco estratégias mediadas por tecnologias, já que os itens descrevem o esclarecimento de dúvidas mediante as ferramentas web: AVA, fóruns e mensagens. Como os alunos da amostra são semipresenciais, o ato de tirar dúvidas possivelmente 
era feito pessoalmente, na sala de aula, em conversas informais nos corredores com colegas ou professores.

A estrutura fatorial do instrumento de Estratégias de Aprendizagem mantém os bons índices de consistência interna e difere empiricamente das encontradas em estudos existentes com a medida (Borges-Ferreira, 2005; Warr \& Downing, 2000; Zerbini e cols., 2005; Zerbini \& Abbad, 2008), sendo adequada ao contexto específico investigado e reafirmando que as evidências de validade estão diretamente ligadas às características do contexto e da amostra. Os oito fatores presentes em Zerbini e Abbad (2008) deram origem a quatro, com rearranjo distinto das diferentes estratégias de aprendizagem - destaque para a junção das estratégias cognitivas em um mesmo fator e permanência de fatores compostos por estratégias autorregulatórias. Como se trata da primeira aplicação do instrumento ao ensino superior híbrido, outros estudos poderão confirmar tal estrutura, que apresenta sentido teórico em função das peculiaridades contextuais.

\section{Considerações finais}

A área de avaliação de cursos no nível superior de ensino, sobretudo cursos de graduação ofertados a distância, é bastante incipiente e carece de estudos sistemáticos, os quais proponham modelos multivariados de avaliação e façam uso de instrumentos de medida específicos à modalidade EAD. Considerando-se que este é um campo de conhecimento ainda em fase de exploração, com poucas pesquisas e instrumentos disponíveis na literatura, conforme observado no levantamento bibliográfico conduzido neste estudo, foram utilizados procedimentos de análises exploratórios para responder aos problemas desta pesquisa.

Dentre as contribuições do estudo estão a adaptação e a validação semântica e por juízes da escala de Estratégias de Aprendizagem, além da verificação de evidências de validade da medida. A escala é composta por 29 itens e apresenta evidências de validade de conteúdo e da estrutura interna, podendo ser utilizada também em futuras pesquisas da área de avaliação de ações educacionais a distância, em organizações de trabalho e instituições de ensino que ofertam cursos EAD. Sugere-se a sua aplicação em contextos e amostras diferentes, sobretudo, em ensino superior essencialmente a distância, além da realização de análise fatorial confirmatória no instrumento.

As limitações dizem respeito principalmente ao baixo índice de devolução dos questionários nos momentos de coleta de dados realizados por meio da internet, através do AVA. Futuras pesquisas em ambientes escolares, especificadamente, do nível superior de ensino, poderão ratificar ou retificar tais dificuldades e aprimorar os meios de coleta de dados. Resultados e discussões proporcionados por pesquisas na área de avaliação de ações educacionais a distância podem auxiliar no convencimento de IES para os benefícios do julgamento de seus programas instrucionais. Assim, ao almejar o retorno da avaliação, as instituições despenderão mais esforços para facilitar e apoiar contundentemente a coleta de dados.

\section{Referências}

Badia, A., \& Monereo, C. (2010). Ensino e aprendizagem de estratégias de aprendizagem em ambientes virtuais. Em C. Coll \& C. Monereo (Orgs.), Psicologia da dducação virtual: aprender e ensinar com as tecnologias da informação e da comunicação. (pp. 311-328). Porto Alegre: Artmed.

Bessa, J., \& Tavares, J. (2001). Níveis de ajustamento e auto-regulação académica em estudantes do $1^{\circ}$ ano (comum) de ciências e engenharias da Universidade de Aveiro. EmJ. Tavares \& R. Santiago (Orgs), Ensino superior. (In)sucesso académico (pp. 107-132). Porto: Porto Editora.

Borges-Ferreira, M. F. (2005). Avaliação de reações e aprendizagem em disciplinas de curso técnico profissionalizante oferecidas a distância. Dissertação de Mestrado, Instituto de Psicologia, Universidade de Brasília.

Boruchovitch, E., \& Santos, A. A. A. (2004). Escala de Avaliação de Estratégias de Aprendizagem para crianças do ensino fundamental. Manuscrito não publicado, Universidade São Francisco, Bragança Paulista.

Boruchovitch, E., Santos, A. A. A., Costa, E. R., Neves, E. R. C., Cruvinel, M., Primi, R., \& Guimarães, S. E. R. (2006). A construção de uma escala de estratégias de aprendizagem para alunos do ensino fundamental. Psicologia: Teoria e Pesquisa, 22(3), 297-304.

Carswell, A. D., \& Venkatesh, V. (2002). Learner outcomes in an asynchronous distance education environment. International Journal of Human-Computer Studies, 56, 475-494.

Castro, M. N. M., \& Ferreira, L. D. V. (2006). TD\&E a distância: múltiplas mídias e clientelas. Em J. E. 
Borges-Andrade, G. Abbad \& L. Mourão (Orgs.), Treinamento, desenvolvimento e educação em organizações e trabalho: fundamentos para a gestão de pessoas (pp. 322339). Porto Alegre: Artmed.

Cheung, D. (2000). Evidence of a single second-order factor in student ratings of teaching effectiveness. Structural Equation Modeling, 7(3), 442-460.

Coll, C., \& Monereo, C. (2010). Educação e aprendizagem no século XXI: novas ferramentas, novos cenários, novas finalidades. Em C. Coll \& C. Monereo (Orgs.), Psicologia da educaşão virtual - aprender e ensinar com as tecnologias da informação e da comunicação (pp. 15-46). Porto Alegre: Artmed.

Costa, E. R., \& Boruchovitch, E. (2000). Fatores que influenciam o uso de estratégias de aprendizagem. Psico-USF, 5, 11-24.

Costa, E. R., \& Boruchovitch, E. (2009). As estratégias de aprendizagem e a produção de textos narrativos. Psicologia: Reflexão e Crítica, 22(2), 173-180.

Laros, J. A. (2004). O uso da análise fatorial: algumas diretrizes para pesquisadores. Em L. Pasquali (Org.), Análise fatorial para pesquisadores. Petrópolis: Vozes.

Levin, J. R. (1986). Four cognitive principles of learning-strategy instruction. Educational Psychologist, 21, 3-17.

Oliveira, K. C., Boruchovitch, E., \& Santos, A. A. A. (2009). Estratégias de aprendizagem e desempenho acadêmico: evidências de validade. Psicologia: Teoria \& Pesquisa, 25(4), 531-536.

Ribeiro, I. S., \& Silva, C. F. (2007). Auto-regulação: diferenças em função do ano e área em alunos universitários. Psicologia: Teoria e Pesquisa, 23(4), 443-448.
Santos, A. A. A., Boruchovitch, E., Primi, R., Zenorini, R. P. C., \& Bueno, J. M. H. (2004). Escala de Avaliação de Estratégias de Aprendizagem para universitários (EAP-U): aplicação do Modelo de Rasch de créditos parciais. Psicologia: Teoria, Investigação e Prática, 9(2), 227-242.

Silva, A. P. (2004). Avaliação de uma disciplina semipresencial de graduação ofertada por meio da internet pela Universidade de Brasília. Dissertação de Mestrado, Instituto de Psicologia, Universidade de Brasília.

Tabachnick, B. G., \& Fidell, L. S. (2001). Using multivariate statistics. Nova Iorque: Harper-Collins College Publishers.

Warr, P., \& Allan, C. (1998). Learning strategies and occupational training. International Review of Industrial and Organizational Psychology, 13, 83-121.

Warr, P., \& Downing, J. (2000). Learning strategies, learning anxiety and knowledge acquisition. British Journal Psychology, 91, 311-333.

Zerbini, T. (2007). Avaliação da transferência de treinamento em curso a distância. Tese de Doutorado, Instituto de Psicologia, Universidade de Brasília.

Zerbini, T., \& Abbad, G. (2008). Estratégias de aprendizagem em curso a distância: validação de uma escala. Psico-USF, 13, 177-187.

Zerbini, T., Carvalho, R. S., \& Abbad, G. (2005). Treinamento a distância via internet: construção e validação de escala de estratégias de aprendizagem [CD-Rom]. Em Associação Nacional dos Programas de Pós-Graduação em Administração (Org.). Anais do XXIX ENANPAD. Brasília: ANPAD.

Recebido em: 06/03/2013

Reformulado em: 03/10/2013

Segunda reformulação em: 19/11/2013

Aprovado em: 05/02/2014 
Sobre as autoras:

Lara Barros Martins é mestre em Psicologia pela Faculdade de Filosofia, Ciências e Letras de Ribeirão Preto - Universidade de São Paulo (FFCLRP/USP), doutoranda do Programa de Pós-Graduação em Psicologia da FFCLRP/ USP, com a realização de um período de investigação na Facultad de Psicología, Universidad de Sevilla. O interesse e foco das pesquisas está na área de Treinamento, Desenvolvimento e Educação de Pessoas (TD\&E) e em avaliação de programas educacionais ofertados a distância.

Thaís Zerbini é doutora e mestre em Psicologia pela Universidade de Brasília, professora doutora em Psicologia Organizacional e do Trabalho da Faculdade de Filosofia, Ciências e Letras de Ribeirão Preto - Universidade de São Paulo (FFCLRP/USP), realiza pesquisas na área de Psicologia e de Administração, com ênfase em Aprendizagem, Treinamento, Desenvolvimento e Educação de Pessoas (TD\&E), Comportamento Organizacional e Gestão de Pessoas.

\section{Contato com as autoras:}

Faculdade de Filosofia, Ciências e Letras de Ribeirão Preto - Universidade de São Paulo.

Departamento de Psicologia

Av. Bandeirantes, 3900 - Monte Alegre

CEP 14040-901 - Ribeirão Preto (SP)

Tel. (16) 3602-4687 